\title{
The Linguistic Features of "Clickbait" in Chinese Websites
}

\author{
Xinyu Lun \\ International College of Macau University of Science and Technology, Macau, China \\ ${ }^{*}$ Corresponding author. Email: 2009853put30001@student.must.edu.mo
}

\begin{abstract}
This article examines the word and sentence characteristics of "clickbait" in Chinese websites. In terms of words, celebrity names, suspenseful words, violent or pornographic words, and exaggerated words are commonly used types in "clickbait." The widely used sentence patterns of "clickbait" include exclamation sentences, omission sentences, interrogative sentences, and rhetorical question sentences. Through the principle of cooperation, these types of words and sentence patterns are to attract readers and increase click-through rates, which is not a good thing for the credibility of the media.
\end{abstract}

Keywords: "clickbait", vocabulary characteristics, sentence feature, the principle of cooperation

\section{INTRODUCTION}

In the 1990s, the Chinese press began to put the news on the Internet, and online news has attracted the public's attention. To a large extent, online news dissemination relies on the guidance and prompts of headlines, so headlines play an extremely prominent role in online dissemination. At the same time, there are many problems with online news headlines. Some online journalists exaggerate or even fabricate facts to attract readers' attention and earn clicks. The "Clickbait" phenomenon is not uncommon, which has aroused widespread concern in academia.

\section{LITERATURE REVIEW}

This article collects relevant literature on "clickbait" from 2011 to 2021 in CNKI and Web of Science. The reason for choosing this period is that these ten years are essential for the emergence and vigorous development of the "clickbait" phenomenon. The research content of these documents mainly includes the characteristics, reasons, and harm of "clickbait."

\subsection{Features}

Qiao(2015) summarized the characteristics of "clickbait" as "(1) exaggerating certain elements of the facts, thereby changing the overall nature of the facts; (2) the title of the text does not match; (3) deliberately setting up the suspense to induce the audience to read;
(4) Fake news, which has not been confirmed or even denied, is reported as facts, using online hot words and popular sentence patterns, so that the audience will have click inertia ${ }^{[1]}$. The "People's Daily" pointed out that "they do not hesitate to use sensationalism, horror, filthy and insulting words and phrases earn attention, and even many serious articles have vulgar titles ${ }^{[2]}$."

\subsection{Reason}

The academic circles also have different opinions on the reasons for the emergence of "clickbait." Xu(2009) argues that the Internet has opened up an unfettered and unrestricted space for anyone to express opinions, coupled with the lack of originality and originality in online news ${ }^{[3]}$. The vulgarization and entertaining tendency of online news are essential reasons for the emergence of "clickbait" news. Huang (2010) believes that online media lacks awareness of "gatekeepers" and regards spoof as a standard, which has also increased the number of "clickbait" in online news ${ }^{[4]}$. Zhu (2012) points out that the misdirection caused by intensified media market competition, the lack of social responsibility of editors and reporters and even the media, and the poor political and professional qualities of editors and reporters are the main reasons for the emergence of "clickbait" ${ }^{\text {[5] }}$.

\subsection{Harm}

The "clickbait" in the news seriously affects the 
media's credibility and misleads and troubles the audience to obtain information accurately. Cao(2013) analyzed the five manifestations of "clickbait" and pointed out that "clickbait" is not a media savior but a news cancer ${ }^{[6]}$. Luo (2015) believes that the prevalence of clickbait has brought severe harm: on the one hand, "clickbait" fabricate facts and distort the truth of the facts, which has created vicious competition among the news industries; on the other hand, it also undermines the credibility of the news media ${ }^{[7]}$. Chen (2014) believes that "clickbait" distorts the value of news and violates news authenticity and objectivity. It is also a manifestation of the lack of professional ethics and moral consciousness in the media ${ }^{[8]}$.

\section{METHODOLOGY}

Clickbait refers to a sensational online news headline made through exaggeration, eroticism, distortion, weirdness, etc ${ }^{[9]}$. According to this definition, 210 "clickbait" titles from 8 representative Chinese news websites ${ }^{[10]}$ are selected. Induction and statistics methods would be used in this research.

\section{DATA ANALYSIS}

"Clickbait" has different characteristics in terms of choice of words and sentence patterns. The following will analyze the linguistic features of "clickbait" from these two perspectives.

\subsection{Choice of Words}

Through TF word frequency ${ }^{[11]}$ statistics on all corpora, it could be found that "clickbait" has a certain commonality in word selection. Celebrity names, suspenseful words, violent or pornographic words, and exaggerated words are the six frequently used words in "clickbait."

\subsubsection{Celebrity Names}

As public social figures, the celebrity group is very special. Their words and deeds have not only attracted the attention of society but may also become the objects of people's pursuit and imitation. Therefore, news headlines with celebrities and related information will naturally attract attention. "Clickbait" is just taking advantage of the high entertainment mood of the whole people to win the click rate. For example,

"Inventory: How rich is Liu Tao, and how many luxury cars does she own!"

\section{(Toutiao)}

"His face is full of blood! Huo Jianhua went to the supermarket without removing his makeup and terrified the crowd."

\section{(Baidu News)}

"Eleven years ago, the girl who "would rather take a $B M W$ to cry instead of a bicycle to laugh", did she marry a rich family?"

(Toutiao)

\subsubsection{Suspenseful Words}

Words such as "revelation," "revealing," and "truth" all contain the meaning of revealing the secret, which could be summarized as suspenseful words. Such words often appear in "clickbait" and are used to express "this news Will solve a certain mystery" to let the reader click on it. For example,

"Attention! The milk you drink may not be milk at all. This is the truth!"

(Toutiao)

"Revealing the truth about XXX's death."

(Sina News)

"Demystify! The truth of the XXX Incident is actually ......"

(Netease News)

\subsubsection{Violent or Pornographic Words}

"Clickbait" often uses words that contain violent or pornographic meanings, which can give readers a frightening feeling and attract readers' attention. For example,

"Counting News of Illicit Love"

(Sohu News)

"The female lawyer was cut off by her husband's domestic violence, and her mother's skull was broken"

(Netease News)

\subsubsection{Exaggerated Words}

To arouse readers' associations and resonance, "clickbait" often uses rich imagination to exaggerate its number, image, influence consciously, etc., based on objective facts, or even fabricate news facts. For example,

"Ferrari can line one kilometer under the name of a local tyrant."

(Netease News)

"Shocked! The man drank 11 tons of liquor and sent him to the hospital..."

(Phoenix News) 


\subsection{Choice of Sentence Pattern}

There are four sentence patterns frequently used in "clickbait": exclamation sentences, omission sentences, interrogative sentences, and rhetorical question sentences.

\subsubsection{Exclamatory Sentence}

Exclamatory sentences often have a solid emotional color, expressing various emotional states such as happiness, surprise, sorrow, disgust, and fear. In "clickbait," exclamatory sentences are often used to describe some uncommon events and express feelings of shock and surprise. For example,

"The liar is crazy! The woman reported bank card information after picking up 280,000 tickets, and the card was only 4 yuan."

(Tencent News)

"Thirty-nine cardiologists are vegetarian! They said: Too many patients have been in contact."

\section{(People's Daily WeChat Public Account)}

"It is a misunderstanding to eat sugar-free moon cakes casually! Experts remind you to eat moon cakes healthily."

(Toutiao)

\subsubsection{Ellipsis}

The omission sentence stimulates readers' interest in reading by setting up suspense and arouses further thinking. For example,

"Shocked! A certain musician organizes a photo contest, and the comparison is actually..."

(Phoenix News)

"The truck is carrying peaches rolls over, but passersby..."

(Phoenix News)

\subsubsection{Interrogative Sentence}

Interrogative sentences arouse readers' attention and thinking by asking questions. For example,

"Has XXX got plastic surgery?"

(Toutiao)

"Did XXX offend the stylist? Why are there so many hairstyles?"

(Toutiao)

"Can licorice soak in water to maintain health? Beware of swelling if you drink too much. "
(Toutiao)

\subsubsection{Rhetorical Question Sentences}

As an interrogative sentence, a rhetorical question sentence is characterized by asking without a doubt, asking and answering by oneself. However, when used in "clickbait," its focus is not on pertinence and enlightenment but a one-sided pursuit of attracting readers' attention, which leads to the abuse of question sentences. For example,

"Do you dare to sit like this when you eat out? The scene captured by surveillance is so scary."

(People's Daily WeChat Public Account)

"Is eating eggs in the morning good or bad for the body? Never expected it."

(People's Daily WeChat Public Account)

\section{DISCUSSION}

Grice believes that in speech activities, both parties in communication have the same desire: both sides can understand each other and cooperate. To better realize this desire, both parties in the communication abide by certain cooperative principles, that is, according to the purpose of the conversation or the direction of communication, the words spoken by themselves are required for communication under certain conditions to realize this desire. The principle of cooperation includes four categories, namely:

- Quantitative criterion: The information contained in the utterance is required to be all the information the speaker wants to say, not exaggerated.

- Qualitative criterion: the words required to be spoken are true.

- Relationship criterion: It is required to have a connection between the spoken words.

- Method criterion: the words spoken are required to be clear and clear.

The new conversational meaning may be produced when the speaker deliberately violates a specific criterion of the cooperative principle. For "clickbait", they often violate qualitative and quantitative guidelines, ignore news facts, exaggerate and distort news content, which gives the audience a one-sided conversational meaning. E.g.:

"Foreign media claim that Chinese people can save the planet and reduce greenhouse gas emissions by eating less meat."

\section{(Sina News)}

The main content of this news is that some 
international environmentalists believe that the latest dietary guidelines issued by the Chinese government are beneficial to public health and environmental protection. Because the new version of the procedures may reduce China's meat consumption or at least slow down its growth rate, thereby helping to save the land and water resources and significantly reduce global greenhouse gas emissions. However, this title directly exaggerates the content, creating the conversational meaning of "eating less meat can save the earth" to the audience and distorting the original words and intentions of the interviewees. Such headlines often ignore the systematic argumentation process of news, impose causality, and mislead readers to believe that certain phenomena will directly lead to specific results, which significantly damages the credibility of news. In addition, "clickbait" also violates the relationship criterion, often the text is not on the topic, and the news content is completely irrelevant or has little relevance to the headline. E.g.:

"The Jinan-Pyongyang route plane used to be a particular plane for Kim Jong-un, and the stewardess spoke Chinese."

\section{(Netease News)}

The purpose of this news is to report that on May 31, 2016, North Korea Pyongyang opened a route. The content has no actual connection with Kim Jong-un, but for the audience, it has the conversational meaning of "related to Kim Jong-un and able to understand Kim Jong-un". The appearance of "Kim Jong Un" in the title attracts readers' attention, causing the title to deviate from the focus of the content. Similarly, most of the words "clickbait" are long and often deliberately cause ambiguity. E.g.:

"He was born in a slum and conquered the fashion circle at the age of 48. Women all over the world want to wear his shoes to get married."

(Phoenix News)

This title has a total of 27 words, and the content tells the story of the founder of Jimmy Choo. "His shoes" has the conversational meaning of "his shoes" to the audience, creating ambiguity. The correct expression should be "shoes made by him" or "shoes of his brand".

In summary, "clickbait" extensively violates the principle of cooperation, making readers often get adequate information related to the topic by reading the article's content. If the public stays in this kind of online news environment for a long time, they will be disgusted and bored to a large extent, reduce their trust in the media, and weaken the media's propaganda.

\section{CONCLUSION}

This article examines the Linguistic features of "clickbait" in Chinese websites through the collection of eight representative Chinese websites and the method of word frequency statistics. In the choice of words, celebrity names, suspenseful words, violent or pornographic words, and exaggerated words are frequently used in "clickbait." In selecting sentence patterns, "clickbait" often uses exclamation sentences, omission sentences, interrogative sentences, and rhetorical question sentences. It could be seen from the principle of cooperation that "clickbait" violates quantitative, qualitative, relationship and method standards, which is not a good thing for the credibility of the media.

\section{REFERENCES}

[1] Qiao Yuanyuan \& Jiang Leiyi. The status and harm of WeChat news "Clickbait". Western Radio and Television. vol. 12, (2015). 29-30. DOI:CNKI:SUN:XBGS.0.2015-12-020.

[2] Xu Miaomiao: "The Language Violence of "Clickbait", "People's Daily", January 23, 2015.

[3] $\mathrm{Xu}$ Wenjing. Analysis of the "Clickbait" phenomenon of online news. Journal of Chongqing Three Gorges University. vol. 01. (2009).109-112. DOI::CNKI:SUN:SCSX.0.2009-01-028.

[4] Huang Zhihong. "Clickbait" Phenomenon in Internet Media. News Frontier. vol.09. (2010). 95-97. DOI:CNKI:SUN: XYQS.0.2010-09-056.

[5] Zhu Jidong. The hazards, root causes and countermeasures of the proliferation of "Clickbait". Journalists. vol. $17 . \quad$ (2012). 15-18. DOI:10.16017/j.cnki.xwahz.2012.17.017.

[6] Cao Lin. "Clickbait" should be discarded in reforms. Leader Wen Cui. vol. 03. (2013). 23-24. DOI:CNKI:SUN: LDWC.0.2013-03-004.

[7] Luo Jia. Research on "Clickbait" news recognition technology based on latent semantic analysis (Master's thesis, Hubei University of Technology). (2015).https://kns-cnkinet.libezproxy.must.edu.mo/ $\mathrm{KCMS} /$ detail/detail.aspx?dbname=CMFD201502\& filename $=1015900621 . \mathrm{nh}$

[8] Chen Lidan. "Clickbait" used the concept of news value to misrepresent. Media. vol. 19. (2014). 8-10. DOI:CNKI:SUN:CMEI.0.2014-19-005.

[9] Wang Chenyao \& Jin Liang. The status quo and narrative strategy of online news "Clickbait"—A quantitative analysis of news rankings of 8 websites. Journalist vol. $\quad 02 . \quad$ (2013). 65-71.DOI:10.16057/j.cnki.31-1171/g2.2013.02.017.

[10] These are Sohu News, Tencent News, Baidu News, Toutiao, People's Daily, Sina News, Netease News, Phoenix News.

[11] TF (Term Frequency) refers to the number of times a given the word appears in the file. 